\title{
Tissue-specific effects of mutations in the thyroid hormone transporter MCT8
}

Simone Kersseboom', Theo J. Visser ${ }^{2}$

$\mathrm{T}$ hyroid hormone $(\mathrm{TH})$ is important for the development of different tissues, in particular the brain, as well as for the regulation of the metabolic activities of the tissues and thermogenesis throughout life. Most TH actions are initiated by binding of the active hormone 3,3',5-triiodothyronine (T3) to its nuclear receptor. This induces an alteration in proteins associated with the transcription initiation complex, resulting in the stimulation or suppression of the expression of $\mathrm{TH}$ responsive genes.

The biological activity of TH is thus determined by the intracellular T3 concentration, which is only indirectly dependent on the function of the thyroid gland which secretes predominantly the prohormone thyroxine (3,3',5, ','tetraiodothyronine, T4). In many target tissues, $\mathrm{T} 3$ availability is regulated in a paracrine manner, where $\mathrm{T} 3$ supply to target cells is derived from T4 to T3 conversion in neighbouring cells. Brain and cochlea are examples of tissues with paracrine regulation of $\mathrm{TH}$ action. In other tissues, such as the pituitary and brown adipose tissue (BAT), T3 may be produced from T4 directly in its target cells, representing an autocrine mechanism of TH action (Figure 1). In yet other tissues such as the liver and the kidneys, intracellular T3 is in rapid exchange with circulating T3. This could be regarded as an endocrine action of T3, although it is still largely derived from peripheral conversion of T4 even in these same tissues.

In contrast to the activation of $\mathrm{T} 4$ by enzymatic outer ring deiodination (ORD) to $\mathrm{T} 3, \mathrm{TH}$ is inactivated by inner ring deiodination (IRD), which converts $\mathrm{T} 4$ to 3,3',5'-triiodothyronine (reverse T3, rT3) and T3 to 3,3'-diiodothyronine (T2) (1). Three iodothyronine deiodinases (Dl-3) are involved in these different deiodination reactions (1). Dl is expressed in liver, kidneys and thyroid and has both ORD and IRD activity. It is thought to contribute importantly to production of serum T3, in particular in eu- and hyperthyroid conditions. D2 has only ORD activity and is expressed in brain, pituitary, BAT, thyroid and skeletal muscle. It is essential for local production of $\mathrm{T} 3$ in brain, pituitary and BAT, but the enzyme in thyroid and muscle may also be an important site for production of serum $\mathrm{T} 3$ in eu- and hypothyroid subjects. D3 is highly expressed in different fetal tissues, placenta and pregnant uterus, and also in adult brain and skin. It has only IRD activity and thus catalyzes the inactivation of TH. Together with D2, D3 has a crucial role in the region-specific and time-dependent regulation of $\mathrm{T} 3$ in the developing brain. The deiodinases are homologous selenoprotein embedded in the membrane of the endoplasmic reticulum or the plasma membrane, such that the active sites are located in the cytoplasm (1).

$\mathrm{TH}$ metabolism and action are intracellular processes that require transport of iodothyronines across the cell membrane. This does not take place by passive diffusion but requires the involvement of specific transporters (2). A number of multi-specific transporters have been identified which are also capable of transporting $\mathrm{TH}$.

1 PhD student, Department of Internal Medicine, Erasmus University Medical Center, Rotterdam, The Netherlands 2 Professor, Department of Internal Medicine, Erasmus University Medical Center, Rotterdam, The Netherlands

Correspondence to:

Theo J. Visser

Department of Internal Medicine, Erasmus MC, Room Ee 502

PO Box 2040, 3000

CA Rotterdam, The Netherlands t.j.visser@erasmusmc.nl 


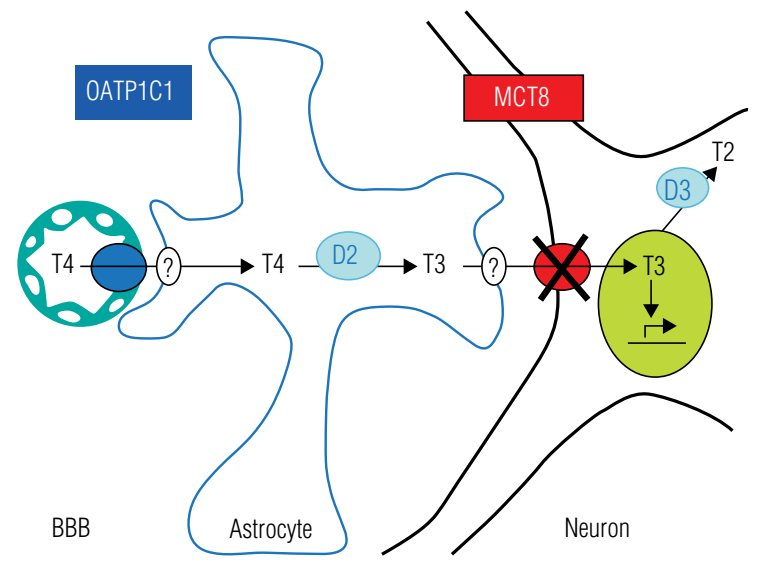

Figure 1. Simplified schema of the regulation of T3 supply to neuronal target cells in brain, and the defect induced by MCT8 mutation.

In addition, three transporters have been characterized showing considerable specificity for iodothyronines, namely OATPlCl, MCT8, and MCTl0 (2). The pathophysiological relevance of $\mathrm{TH}$ transporters has become clear in particular for MCT8, mutations in which are the cause of a syndrome of X-linked psychomotor retardation combined with abnormal TH levels $(3,4)$. The clinical characteristics of a patient with a novel mutation in MCT8 are described by de Menezes Filho and cols. (5).

\section{MOLECULAR CHARACTERISTICS OF MCT8}

MCT8 and MCT10 are members of what is called the monocarboxylate transporter (MCT) family, although only 4 members of this family (MCTl-4) are known to transport monocarboxylates such as lactate and pyruvate (6). The function of most of the 14 members of this family is still enigmatic. In 2001, MCT10 was identified as an aromatic amino acid transporter and we have subsequently demonstrated that MCT8 and MCTI0 are effective $\mathrm{TH}$ transporters (2).

The MCT8 gene is located on human chr Xq13.2 and has 6 exons and, thus, 5 introns of which intron 1 is $\sim 100 \mathrm{~kb}$ in size. MCT8 has two possible translation start sites (TLSs), yielding proteins of 613 or 539 amino acids. In many animals MCT8 lacks the first TLS giving rise to only the short MCT8 protein. If the N-terminal extension of the long human MCT8 protein has specific functions remains to be determined. The MCT10 gene has a very similar structure, is located on human chr 6q21-q22, and codes for a protein of 515 amino acids. In all species, MCT10 has only one TLS, corresponding to the second TLS of human MCT8.
MCT8 and MCT10 have 12 putative transmembrane domains (TMDs), with both $\mathrm{N}$-and C-terminal domains located intracellularly. MCT8 and MCT10 show a high degree of homology, especially in their TMDs, which fits with their similar functions as TH transporters. Both MCT8 and MCT10 transport different iodothyronines; $\mathrm{T} 3$ is transported somewhat better by MCT10 than by MCT8, whereas the opposite is true for T4 (2). Both MCT8 and MCT10 facilitate cellular uptake as well as efflux of iodothyronines. Expression of MCT8 or MCT10 may thus induce only a modest increase in steady-state intracellular TH levels. However, transfection of cells with MCT8 or MCT10 strongly increases iodothyronine metabolism by D1, D2 or D3-expressing cells, indicating that they indeed effectively increase intracellular TH availability (2). Prevention of T4 and T3 efflux by co-transfection with the high-affinity cytoplasmic TH-binding protein CRYM, allows the proper study of TH uptake facilitated by MCT8 and MCT10 (2). TH efflux by MCT8 and MCT10 has been less well studied.

MCT8 and MCT10 are expressed in many human tissues. MCT8 is highly expressed in liver, kidney, adrenal, ovary and thyroid. Studies in mice have shown that MCT8 is also expressed importantly in brain, in particular in neurons in different brain regions, including cerebral cortex and cerebellum, but also in the choroid plexus, in capillaries and in tanycytes lining the $3^{\text {rd }}$ ventricle (7). MCT8 has been localized in different nuclei in the human hypothalamus and in the human pituitary (8), where it appears to be expressed predominantly apparently in folliculostellate cells.

\section{PATIENTS}

Mutations in MCT8 are associated with X-linked psychomotor retardation and abnormal serum TH levels $(2-4)$. The neurological syndrome is also known as the Allan-Herndon-Dudley syndrome (AHDS) after the authors of the first study of a large family with affected males published in 1944. AHDS comprises central hypotonia associated with poor head control and initially also peripheral hypotonia that progresses to hypertonia and spasticity. Most AHDS patients are unable to sit, stand or walk independently and have not developed speech. However, in some families patients have a milder phenotype and are able to walk and/or talk, albeit with great difficulty. All patients have severe mental retardation. AHDS patients are born without 
apparent abnormality and the disease appears to develop progressively, often associated with microcephaly. MRI of the brain usually shows delayed myelination before the age of 2 years which apparently normalizes with increasing age. For a more detailed description of the clinical characteristics, readers are referred to Holden and cols. (9) and the paper by de Menezes Filho and cols. (5).

In addition to the psychomotor retardation, AHDS patients have abnormal serum TH levels. Serum T4 and FT4 levels vary from low-normal to truly reduced, whereas serum T3 levels are invariably increased. Like $\mathrm{T} 4$, serum rT3 is often decreased, and the serum T3/ rT3 and T3/T4 ratios are markedly elevated. Serum TSH varies between normal and elevated; mean TSH levels are about twice the normal mean. In view of the low (F)T4 and somewhat higher TSH levels, many AHDS patients have been treated with $\mathrm{T} 4$ substitution without any obvious benefit. This is also the case with the patient reported by de Menezes Filho and cols. (5).

$\mathrm{TH}$ is crucial for brain development. In AHDS patients, brain development is impaired despite the presence of elevated serum T3 levels, indicating some form of $\mathrm{TH}$ resistance. This is explained by the lack of T3 uptake in neuronal target cells where T3 action is required for optimal brain development (Figure 1). AHDS only occurs in males since the MCT8 gene is located on the $\mathrm{X}$ chromosome. However, one female has been described with AHDS due to chromosomal translocation with a breakpoint in MCT8 on one $\mathrm{X}$ chromosome and unfavourable inactivation of the intact X chromosome (10).

In over 50 families with AHDS studied so far various mutations in MCT8 have been identified. These mutations include large deletions, frame-shift mutations and non-sense mutations, which obviously inactivate MCT8. However, for mutations resulting in the deletion, insertion or substitution of single amino acids this is less obvious. The consequences of these mutations have been tested in cells transfected with wild-type (wt) or mutant MCT8 alone or in combination with cDNA coding for D3. These cells have subsequently been tested for TH uptake or TH metabolism, respectively. All tested mutations result in a marked decrease in $\mathrm{TH}$ transport, although the magnitude of the defect depends on the cell type used for transfection $(2,11)$. The results suggest some correlation between the severity of the clinical phenotype, the changes in serum TH levels and the defect in $\mathrm{TH}$ transport.

\section{TISSUE AND HORMONE-SPECIFIC EFFECTS OF MCT8 INACTIVATION}

Inactivation of MCT8 appears to have different effects on TH availability in different tissues. As mentioned above, the neurological phenotype of patients with MCT8 mutations is explained by the lack of T3 uptake in central neurons. This appears to be supported by observations that brain T3 uptake is almost completely blocked in Mct8 knockout (ko) mice, whereas brain T4 uptake is not affected (12). However, brain T3 uptake is probably largely determined by transport across the blood-brain barrier (BBB) and/or the blood-CSF barrier (BCB) and indeed MCT8 is importantly expressed in brain capillaries and in the choroid plexus $(7,13)$. The lack of effect of Mct8 inactivation on brain uptake of T4, which is also a substrate for MCT8, may be explained by assuming that T4 is largely transported across the BBB and BCF by OATPICl $(7,13)$.

In contrast to the subnormal T3 levels in brain, tissue T3 levels may be normal or even increased in other tissues, depending on the repertoire of $\mathrm{TH}$ transporters expressed. Although MCT8 is highly expressed in liver, hepatic T4 and T3 uptake do not seem to be affected in Mct8 ko mice (12). As the liver is exposed to increased serum T3 levels, there is increased expression of T3 responsive genes such as Diol in the liver of Mct8 ko mice (12). This is in agreement with the elevated serum SHBG levels in AHDS patients, knowing that hepatic expression of SHBG is stimulated by $\mathrm{T} 3$ and increased in patients with hyperthyroidism (14). The body composition of AHDS patients also suggest increased metabolic rate and muscle wasting due to tissue hyperthyroidism. This is also reported in the present study by de Menezes Filho and cols (5). Also tachycardia has been observed in AHDS patients.

Mct8 ko have been generated to study the pathogenic mechanism of AHDS, but disappointingly these mice do not show any obvious neurological phenotype $(12,15)$. However, they do show the same changes in serum TH levels as AHDS patients, including a markedly decreased serum T4 and a markedly increased serum T3. Studies into the mechanisms of these alterations have indicated a) increased Dl expression in liver and kidney, b) increased D2 activity in brain and pituitary, and c) decreased D3 activity in brain of Mct8 ko vs. wt animals $(12,16)$.

In contrast to the impaired brain $\mathrm{T} 3$ transport and unaffected hepatic T4 and T3 transport, renal T4 and 
T3 accumulation is increased in the kidneys of Mct8 ko vs. wt mice $(12,16)$. This paradoxical change may suggest that MCT8 is more important for TH efflux than for $\mathrm{TH}$ uptake in the kidney. The increased tissue $\mathrm{T} 4$ content together with the high renal Dl activity may contribute to the large increase in peripheral T4 to T3 conversion, and thus, to the low T4 and high serum T3 levels in Mct8 ko mice and AHDS patients (16).

Recent studies have unveiled an important function of MCT8 also in TH secretion $(17,18)$. Thyroidal T4 and T3 content is increased in Mct8 ko vs. wt mice. Furthermore, the increase in serum T4 induced by TSH injection is lower, whereas the increase in serum $\mathrm{T} 3$ is higher in Mct8 ko vs. wt mice (18). This suggests that MCT8 plays an important role in T4 secretion by thyroid cells. If MCT8 is inactivated, T4 accumulates in the thyrocyte leading to increased intra-thyroidal T4 to $\mathrm{T} 3$ conversion, and thus an increase in the $\mathrm{T} 3 / \mathrm{T} 4$ secretion ratio.

Finally, MCT8 is expressed in both the hypothalamus and the pituitary, and its inactivation may thus interfere with the negative feedback of $\mathrm{TH}$ at both sites $(12,18)$. Indeed, serum TSH is usually modestly increased in AHDS patients, which may be compatible with the modestly decreased serum T4 levels but not with the strongly elevated serum T3 levels. In Mct8 ko mice hypothalamic TRH expression is markedly increased which can only be suppressed by very high T3 doses, indicating $\mathrm{T} 3$ resistance particularly at the hypothalamic level.

The study by de Menezes Filho and cols. (5) reported in this issue presents important information regarding $\mathrm{TH}$ dysregulation in AHDS patients. In agree- ment with previous studies, their patient exhibits low serum FT4 and elevated T3 and TSH levels, in agreement with an impaired negative feedback of T3. Because of the decreased T4 and increased TSH, the patient was treated with LT4. This resulted in a decrease in serum TSH and, remarkably, also a decrease in serum T3. Plotting their data indicates that serum TSH shows a remarkably strong negative correlation with serum FT4 and an equally strong positive correlation with serum T3 (Figure 2). These results are compatible with the view that thyroidal $\mathrm{T} 3$ secretion may be an important source of serum T3 in AHDS patients.

\section{TREATMENT}

The lack of a neurological phenotype in Mct8 ko mice precludes their use as a model for development of an effective treatment of AHDS patients. The possible success of such a treatment will depend on a) the extent to which irreversible damage in brain development has occurred at the time the disease is diagnosed, and b) the possibility to supply active hormone to important brain targets in the absence of functional MCT8. $\mathrm{TH}$ is essential for prenatal brain development, but $\mathrm{TH}$-dependent brain maturation continues after birth (19). The lack of obvious abnormalities at birth and the progressive nature of AHDS may suggest that early postnatal initiation of an effective therapy may have substantial beneficial effect.

Because of the low serum T4 in combination with a modestly increased serum TSH, many AHDS patients have been treated with LT4 without obvious benefit. In fact, such treatment may have detrimental effects as
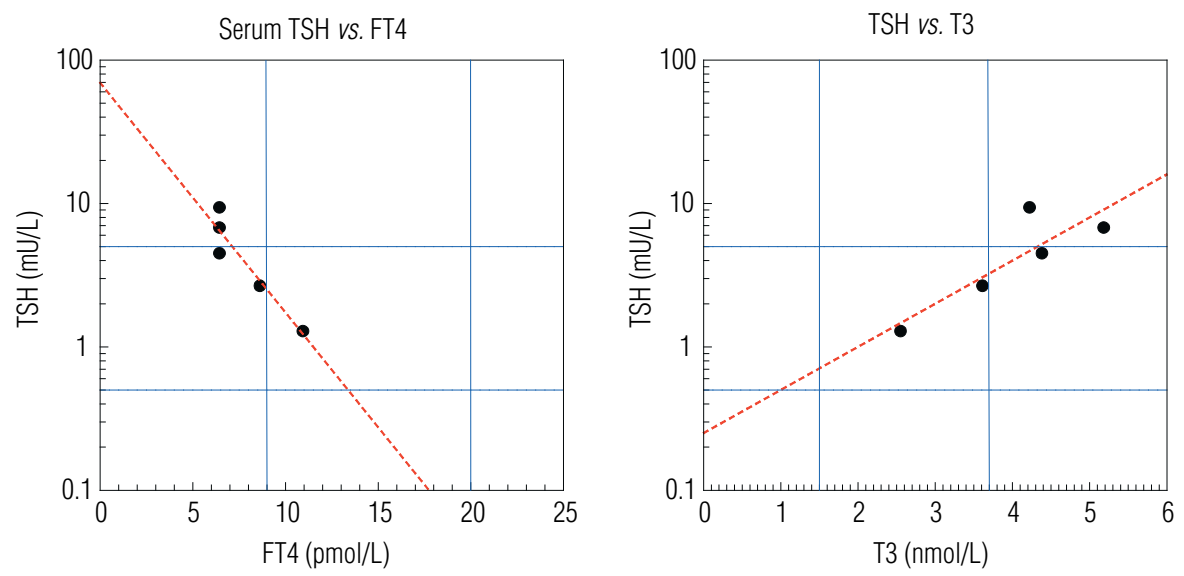

Figure 2. Plots of serum TSH vs. FT4 (left) or T3 (right) using data reported by de Menzed Filho and cols. (5). 
it may further increase T3 exposure of tissues which do not depend on MCT8 for TH uptake. For instance, it may induce or aggravate tachycardia. It could also be hypothesized that the abnormal serum $\mathrm{T} 4$ and $\mathrm{T} 3$ levels contribute importantly to the clinical phenotype and that their normalization may have beneficial therapeutic effects. Such a study has been carried out in an adolescent AHDS patient in whom serum TH levels were normalized by block-and-replace therapy with PTU plus LT4. This produced favourable effects on body weight and heart rate but - as expected - did not result in an obvious mental improvement (14).

Obvious possibilities for effective therapy include early treatment of AHDS patients with a thyromimetic which is effectively transported into the brain even if MCT8 is mutated. The T3 analogue DITPA is currently tested clinically in MCT8 patients (20). Preclinical studies are also in progress to test if the bioactive $\mathrm{TH}$ metabolite Triac or its precursor Tetrac may be used for effective treatment of AHDS patients.

Acknowledgment: Simone Kersseboom is supported by the Smile Foundation

\section{REFERENCES}

1. Gereben B, Zavacki AM, Ribich S, Kim BW, Huang SA, Simonides WS, et al. Cellular and molecular basis of deiodinase-regulated thyroid hormone signaling. Endocr Rev. 2008;29:898-938.

2. Visser WE, Friesema EC, Visser TJ. Minireview: thyroid hormone transporters: the knowns and the unknowns. Mol Endocrinol. 2011;25:1-14

3. Dumitrescu AM, Liao XH, BestTB, Brockmann K, Refetoff S. A novel syndrome combining thyroid and neurological abnormalities is associated with mutations in a monocarboxylate transporter gene. Am J Hum Genet. 2004;74:168-75.

4. Friesema EC, Grueters A, Biebermann H, Krude H, von Moers A, Reeser $\mathrm{M}$, et al. Association between mutations in a thyroid hormone transporter and severe $\mathrm{X}$-linked psychomotor retardation. Lancet. 2004;364:1435-7.

5. Menezes Filho HC, Marui S, Manna TD, Brust ES, Radonsky V, Kuperman $\mathrm{H}$, et al. Novel mutation in MCT8 gene in a Brazilian boy with thyroid hormone resistance and severe neurologic abnormalities. Arq Bras Endocrinol Metab. 2011;55(1):60-6.
6. Halestrap AP, Meredith D. The SLC16 gene family-from monocarboxylate transporters (MCTs) to aromatic amino acid transporters and beyond. Pflugers Arch. 2004;447:619-28.

7. Heuer H, Maier MK, Iden S, Mittag J, Friesema EC, VisserTJ, et al. The monocarboxylate transporter 8 linked to human psychomotor retardation is highly expressed in thyroid hormone-sensitive neuron populations. Endocrinology. 2005;146:1701-6.

8. Fliers $E$, Unmehopa UA, Alkemade A. Functional neuroanatomy of thyroid hormone feedback in the human hypothalamus and pituitary gland. Mol Cell Endocrinol. 2006;251:1-8

9. Holden KR, Zuniga OF, May MM, Su H, Molinero MR, Rogers RC, et al. X-linked MCT8 gene mutations: characterization of the pediatric neurologic phenotype. J Child Neurol. 2005;20:852-7.

10. Frints $S G$, Lenzner $S$, Bauters $M$, Jensen LR, Van Esch $H$, des Portes $\mathrm{V}$, et al. МCT8 mutation analysis and identification of the first female with Allan-Herndon-Dudley syndrome due to loss of MCT8 expression. Eur J Hum Genet. 2008;16:1029-37.

11. Kinne A, Roth S, Biebermann H, Koehrle J, Gruters A, Schweizer $\mathrm{U}$. Surface translocation and T3 uptake of mutant MCT8 proteins are cell type-dependent. J Mol Endocrinol. 2009;43:263-71.

12. Trajkovic M, VisserTJ, Mittag J, Horn S, Lukas J, Darras VM, et al. Abnormal thyroid hormone metabolism in mice lacking the monocarboxylate transporter 8. J Clin Invest. 2007;117:627-35.

13. Roberts LM, Woodford K, Zhou M, Black DS, Haggerty JE, Tate $\mathrm{EH}$, et al. Expression of the thyroid hormone transporters MCT8 (SLC16A2) and OATP14 (SLCO1C1) at the blood-brain barrier. Endocrinology. 2008;149:6251-61.

14. Wemeau JL, Pigeyre M, Proust-Lemoine E, d'Herbomez M, Gottrand $F$, Jansen J, et al. Beneficial effects of propylthiouracil plus L-thyroxine treatment in a patient with a mutation in MCT8. J Clin Endocrinol Metab. 2008;93:2084-88.

15. Dumitrescu AM, Liao XH, Weiss RE, Millen K, Refetoff S. Tissue-specific thyroid hormone deprivation and excess in monocarboxylate transporter (mct) 8-deficient mice. Endocrinology. 2006;147:4036-43.

16. Trajkovic M, VisserTJ, Darras WM, Friesema ECH, Schlott B, Mittag J, et al. Consequences of MCT8 deficiency for renal transport and metabolism of thyroid hormones in mice. Endocrinology. 2009;151:802-9.

17. Di Cosmo C, Liao XH, Dumitrescu AM, Philp NJ, Weiss RE, Refetoff $\mathrm{S}$. Mice deficient in MCT8 reveal a mechanism regulating thyroid hormone secretion. J Clin Invest. 2010;120:3377-88.

18. Trajkovic-Arsic M, Müller J, Darras VM, Groba C, Lee S, Weih D, et al. Impact of monocarboxylate transporter (Mct)-8 deficiency on the hypothalamus-pituitary-thyroid axis in mice. Endocrinology. 2010;151(10)5053-62.

19. Bernal J. Thyroid hormone receptors in brain development and function. Nat Clin Pract. 2007;3:249-59.

20. Di Cosmo C, Liao XH, Dumitrescu AM, Weiss RE, Refetoff $S$. A thyroid hormone analog with reduced dependence on the monocarboxylate transporter 8 for tissue transport. Endocrinology. 2009:150:4450-8. 